\title{
Revision Total Hip Arthroplasty in Osteogenesis Imperfecta
}

\author{
Lauren H. Schoof, BS ${ }^{1}$ Matthew L. Duenes, BS ${ }^{1} \quad$ James E. Feng, MD, MS ${ }^{1,2}$ William J. Long, MD, FRCSC ${ }^{1}$ \\ Ran Schwarzkopf, MD, MSc ${ }^{1}$
}

${ }^{1}$ Department of Orthopedic Surgery, NYU Langone Orthopedic Hospital, NYU Langone Health, New York, New York

${ }^{2}$ Department of Orthopaedic Surgery, Beaumont Health, Royal Oak, Michigan

Address for correspondence Ran Schwarzkopf, MD, MSc, Department of Orthopedic Surgery, NYU Langone Orthopedic Hospital, NYU Langone Health, 301 East 17th Street, New York, NY 10003 (e-mail: schwarzk@gmail.com).

J Hip Surg 2020;4:94-100.

\begin{abstract}
Keywords

- osteogenesis imperfecta

- revision hip arthroplasty

- bone fragility

- complex total hip arthroplasty

Osteogenesis imperfecta (OI) is a rare group of connective tissue disorders commonly associated with bone fragility, deformity, and fractures, leading to instability and early osteoarthritis. Current advancements in the medical and surgical management of $\mathrm{OI}$ have extended the life span of these patients, resulting in an increased need for joint arthroplasty to help maintain quality of life. However, surgery among this patient population is technically challenging and comes with a high burden of revision. In this report, we present a case of revision total hip arthroplasty in a patient with OI and review important considerations to help ensure proper preoperative planning and correct precautions to minimize complications and decrease implant failure.
\end{abstract}

Osteogenesis imperfecta (OI) is a group of autosomal dominant collagen type I (COL1A1 and COL1A2) connective tissue disorders commonly associated with bone fragility, deformity, and fractures. ${ }^{1,2}$ Other common manifestations of OI include blue or gray sclera, dentinogenesis imperfecta, joint hypermobility, hearing impairment, and cardiovascular and central nervous system complications. ${ }^{1-3}$ While certain genetic variants of $\mathrm{OI}$ are intrauterine lethal, others can have life expectancies comparable with that of the general population. ${ }^{4,5}$ Furthermore, advancements in medical and surgical treatments for OI have only enhanced their longevity and quality of life. ${ }^{6}$ However, with their increasing life expectancy, a growing population of ambulatory OI patients is presenting for the treatment of endstage osteoarthritis in their hips and knees. ${ }^{7}$

Primary total hip arthroplasty (THA) is challenging in patients with OI due to poor bone quality, anatomical deformities, and soft tissue laxity. Additionally, with a prevalence between 0.3 and 0.8 per 10,000 births, these patients present as rare and technically challenging surgical THA candidates. ${ }^{1,8-10}$ In Krishnan et al's 2013 case series of six OI patients undergoing primary THA, THA survival rates were reported to

received

June 15, 2019

accepted after revision

March 4, 2020

published online

June 11, 2020 be only $16 \%$ (1/6) at a median time of 5.2 years (range: $2.8-11$ years), demonstrating the high revision burden of these patients. ${ }^{11}$ Given that revision surgery comes with more frequently encountered anatomical deformities and excessive bone loss, it is imperative to understand the outcomes associated with revision THA to further inform the surgical management of patients with OI. Very few cases of revision THA in adult patients with OI have been reported to date. Here we present a report on a patient with OI undergoing a second revision THA.

\section{Case History}

A 62-year-old male with a history of OI presented to our clinic for the evaluation of right hip pain localized to the groin for the past 5 months. He initially underwent right primary THA 22 years ago for symptomatic osteoarthritis. This was revised 14 years later secondary to pain from aseptic failure. The patient had improved pain until approximately 5 months prior to the presentation, when he began having start-up pain in the right hip and worsening pain with ambulation. The pain was

Copyright $\odot 2020$ by Thieme Medical Publishers, Inc., 333 Seventh Avenue, New York, NY 10001, USA

DOI https://doi.org/ 10.1055/s-0040-1712521. ISSN 2472-8446. 
managed conservatively with ambulatory assist devices, physical therapy, and anti-inflammatory medication. However, he reported continued deterioration. At the time, he ambulated with a cane for five blocks, avoided going up and down stairs, was unable to use public transportation, and had difficulty reaching down to put on his right sock and shoe. He did not smoke and had less than one drink per week. His history was also significant for severe scoliosis and multiple fractures that required surgical intervention. Most recently, the patient suffered a left wrist fracture requiring open reduction internal fixation (ORIF). He has also undergone ORIF for a left hip fracture more than 30 years ago and required surgery on his bilateral elbows, right femur, and left shoulder. His OI was previously managed with ibandronate, but he has discontinued the medication for some time. The remainder of his medical history is unremarkable.

\section{Physical Examination and Radiographic Assessment} On physical examination, the patient weighed $71 \mathrm{~kg}$ with a body mass index of $25.2 \mathrm{~kg} / \mathrm{m}^{2}$. He ambulated with the assistance of a cane and had an antalgic gait. A well-healed surgical scar was present over the patient's right hip. He endorsed tenderness to palpation over the right greater trochanter. Range of motion of the right hip was limited to 60 degrees of forward flexion, 20 degrees of external rotation, 10 degrees of internal rotation, 10 degrees of abduction, and 10 degrees of adduction. There was no leg length discrepancy noted on clinical examination. Radiographic imaging of the right hip demonstrated a loose cemented right THA in varus alignment with evidence of protrusion through the lateral femoral cortex (-Fig. 1). Substantial proximal femur remodeling and heterotopic ossification were also visualized. The patient was referred from an outside institute where an infection work-up was conducted, including C-reactive protein and erythrocyte sedimentation rate, and the results were conveyed to us prior to the patient's preliminary visit. After extensive discussion regarding risks and benefits of the surgery, the patient elected to proceed with right revision THA.

\section{Operative Intervention}

After induction of general anesthesia, the patient was turned to the lateral decubitus position. A modified Kocher-Langenbeck incision was made, and the soft tissue was dissected to expose the implant. Intraoperative examination was significant for a loose femoral stem. Using the same implant system, the acetabular liner was exchanged and upsized from $32 \mathrm{~mm}$ to $36 \mathrm{~mm}$ with an elevated lip (StelKast). The $58-\mathrm{mm}$ cup from the prior revision was retained due to the low quality of bone. After the stem was safely extracted without fracture, a prophylactic wire was placed distally to prevent fracturing of the femur while reaming. Cement was removed safely from the canal with the help of cement removal tools and osteotomes (Moreland set, DePuy). Fluoroscopic imaging was used to assess placement of the reamer in the proper canal and confirmed that no disruption occurred.

Due to severe deformity of the femur, an osteotomy was performed and the femur was realigned over the long stem that was placed. After successful trial reduction, the final stem, a 300-mm titanium tapered fluted monoblock stem (REDAPT, Smith \& Nephew) with high offset and a 36-mm femoral head, was placed. A second cable was placed distally, and multiple no. 5 Fiberwires (Arthrex) were passed to secure the osteotomy. The hip was reduced, taken through range of motion, and found to be stable. Intraoperative X-rays were obtained and reviewed prior to closure of the wound. No fractures were visualized, and all implants were in an acceptable position. The remainder of the surgery proceeded in standard fashion and without further complications. While we routinely take an intraoperative aspiration for cell count and differential, in this case we could not obtain intraoperative joint fluid.

The patient was mobilized with physical therapy with $50 \%$ partial weight-bearing on postoperative day 1 . His hospital course was otherwise uncomplicated, and he was discharged home on postoperative day 4 .

\section{Postoperative Care}

At the patient's first postoperative visit, radiographs demonstrated that the implant remained in a good position without evidence of loosening (-Fig. 2). He was cleared for full weight-bearing as tolerated 6 weeks postoperatively. By 3 months, the patient was ambulating independently without an assistive device. Forward flexion of the hip was improved to 90 degrees. Follow-up standard radiographs demonstrated a well-fixed, well-positioned right THA. At his most recent follow-up, 14 months postoperatively, the patient had no complaints regarding the right hip and he continued to ambulate freely ( - Fig. $\mathbf{3}$ ).

\section{Discussion}

Given the incidence of posttraumatic and osteoarthritis in adults with OI, an increasing population of patients who fail nonsurgical interventions are electing to undergo surgical management for their arthritis. However, in this patient group, the complication rate associated with hip arthroplasty is substantially higher than that of the general population, and implant longevity is diminished. ${ }^{12}$ Therefore, prior to surgical intervention, it is imperative that several risk factors are considered, including the quality of the underlying bone, the alignment of the preoperative limb, and any abnormal pelvic or femoral anatomy. Further understanding of which patients require revision surgery can also help inform surgical planning and the management of this complex population, as well as providing for an adequate fund of knowledge to appropriately manage patient expectations.

One anatomical defect, protrusio acetabuli, was found to be significantly associated with THA revision and is commonly observed in 29 to $66 \%$ of patients with OI. ${ }^{11,13,14}$ In a case series of six hips with OI by Krishnan et al., 83.3\% (5/6) of primary THAs required revisions. ${ }^{11}$ Among this group, patients with preoperative acetabular protrusio were significantly more likely to be revised than those without. This study also found a greater mean number of revisions among patients with a prior femoral or acetabular fracture compared with those 


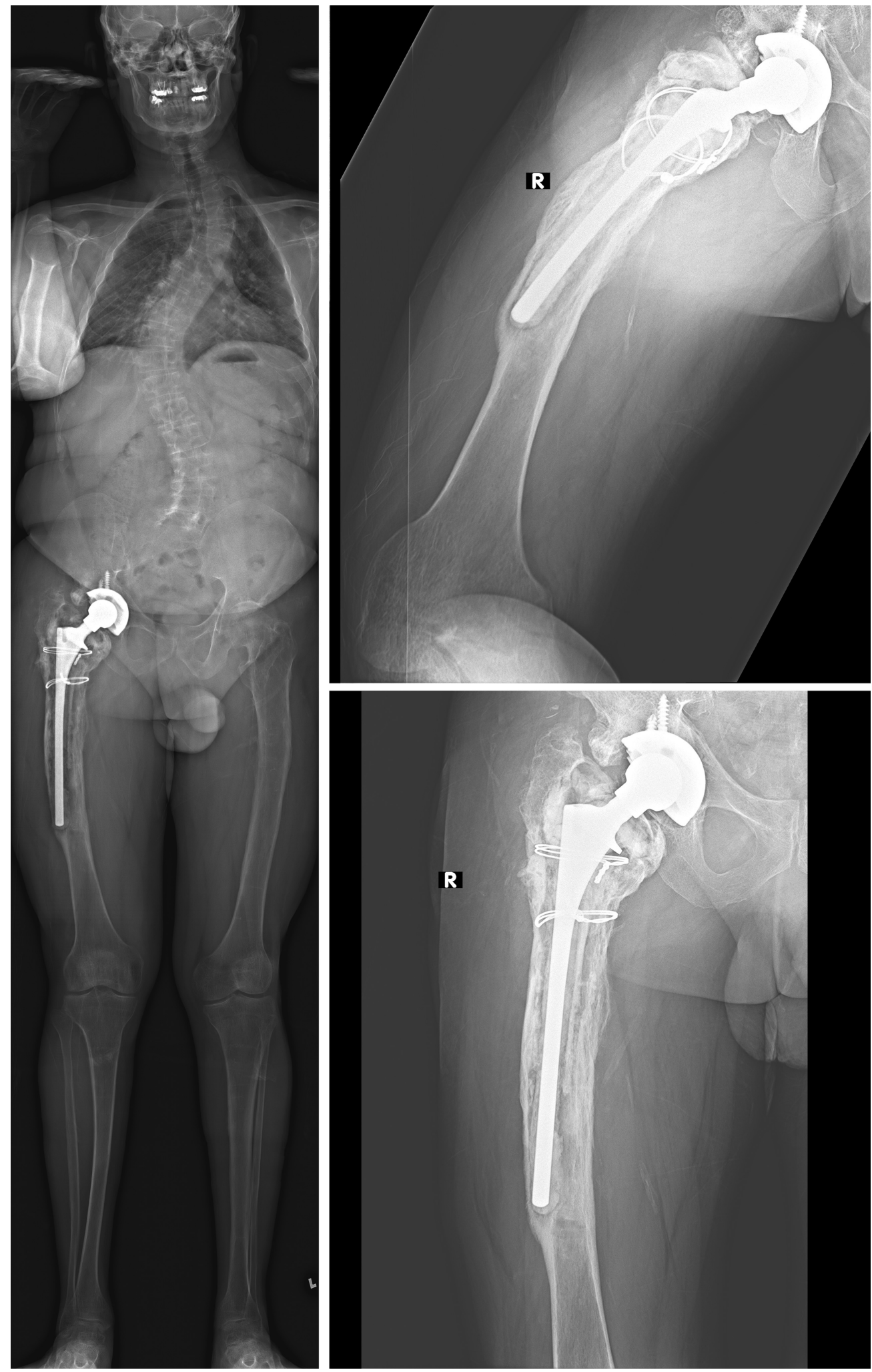

Fig. 1 Preoperative imaging. Anteroposterior, lateral, and lower extremity radiographs demonstrating a previous revision total hip arthroplasty with distal stem migration into a pseudocanal.

without. Papagelopoulos and Morrey also described a series of five THAs, and although only one patient in this study had a reported failure, the patient also had protrusio. ${ }^{7}$ While this anatomical defect is less commonly seen in patients without metabolic bone disease, protrusio acetabuli can be secondary to inflammatory causes, seen in up to $15 \%$ of patients with rheumatoid arthritis of the hip and 33\% of patients with ankylosing spondylitis. It is also estimated to occur in approximately $5 \%$ of patients with osteoarthritis. ${ }^{15}$ However, survivorship of primary THA implants in these patients is reported 

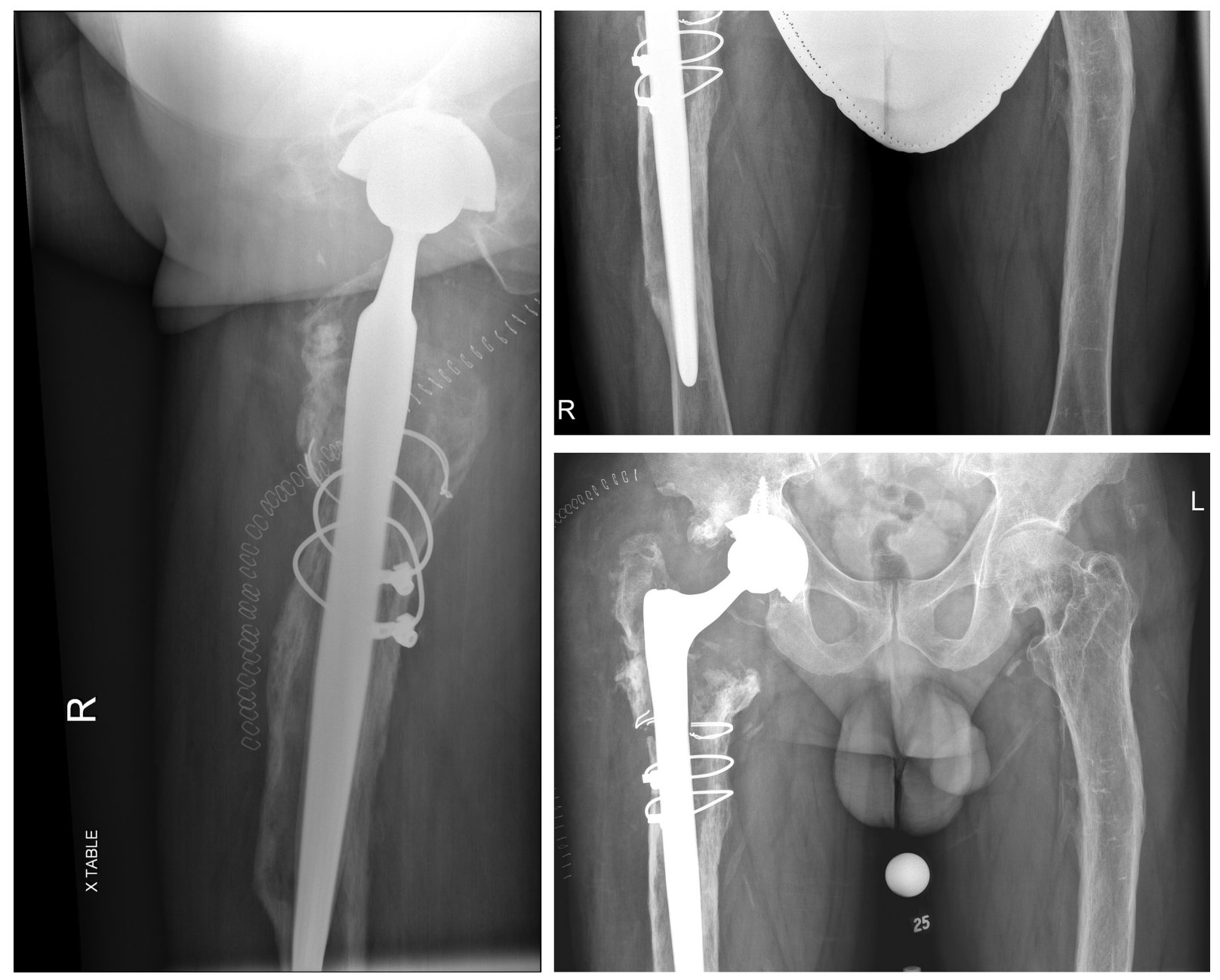

Fig. 2 Three-week postoperative imaging. Anteroposterior pelvis and cross-table radiographs demonstrating a well-fixed, well-positioned right total hip arthroplasty.

between 73 and $100 \%$, with a maximum follow-up of 20 years. ${ }^{16}$ While no large studies have been conducted on the rate of revision THA in patients with OI and protrusio, current literature suggests an increased failure rate among these patients. Although the presence of confounding variables makes it more difficult to reach a definite conclusion, acetabular protrusion adds complexity to an already technically challenging operation and is important to consider preoperatively, if present. In our case, the patient did not have acetabular protrusio but did have a remote history of a right femoral fracture and two prior arthroplasty surgeries. The patient's poor bone quality and prior revision surgery, known risk factors for aseptic loosening, likely also contributed to this second failure.

Another important consideration prior to surgical intervention is intraoperative fracture, with rates as high as $50 \%$ reported in OI patients undergoing primary THA. ${ }^{11} \mathrm{~A}$ larger study of osteoporotic patients found the intraoperative fracture rate to be approximately $4 \%$ after hip replacement, with even higher rates reported in revision surgery. ${ }^{17-19}$ This would suggest a similarly increased risk in patients with OI who have poor bone quality in addition to other anatomical defects, though no studies have demonstrated this effect.
These studies also show, in the general population, that risk factors for intraoperative fracture in THA include osteoporosis, revision surgery, stem length, low ratio between the cortical and canal diameters, metabolic bone disease, and the use of noncemented, press-fit implants.

There is no general agreement on the most appropriate type of implant to minimize the incidence of intraoperative fracture, bone loss, and implant failure among this high-risk population. Papagelopoulos and Morrey reported satisfactory results with cemented implants, with only one patient requiring revision and no report of intraoperative fracture. ${ }^{7}$ Krishnan et al used only noncemented stems in primary surgery, resulting in 50\% incidence of intraoperative fracture and $16 \%$ survival rate of the implant in primary THA at a median time of 5.2 years. However, the authors suggested the use of customized femoral components to minimize fracture in patients with narrow canals and thin cortices. ${ }^{11}$ Among the general population, the highest rate of intraoperative fracture is reported at $20.9 \%$ in patients undergoing revision THA with noncemented implants compared with $3.6 \%$ in revision THA with cemented implants. ${ }^{18}$ Additional data from a Scandinavian arthroplasty registry showed that in patients with osteoporosis, there was an 8.8 times increased risk of early periprosthetic fractures 

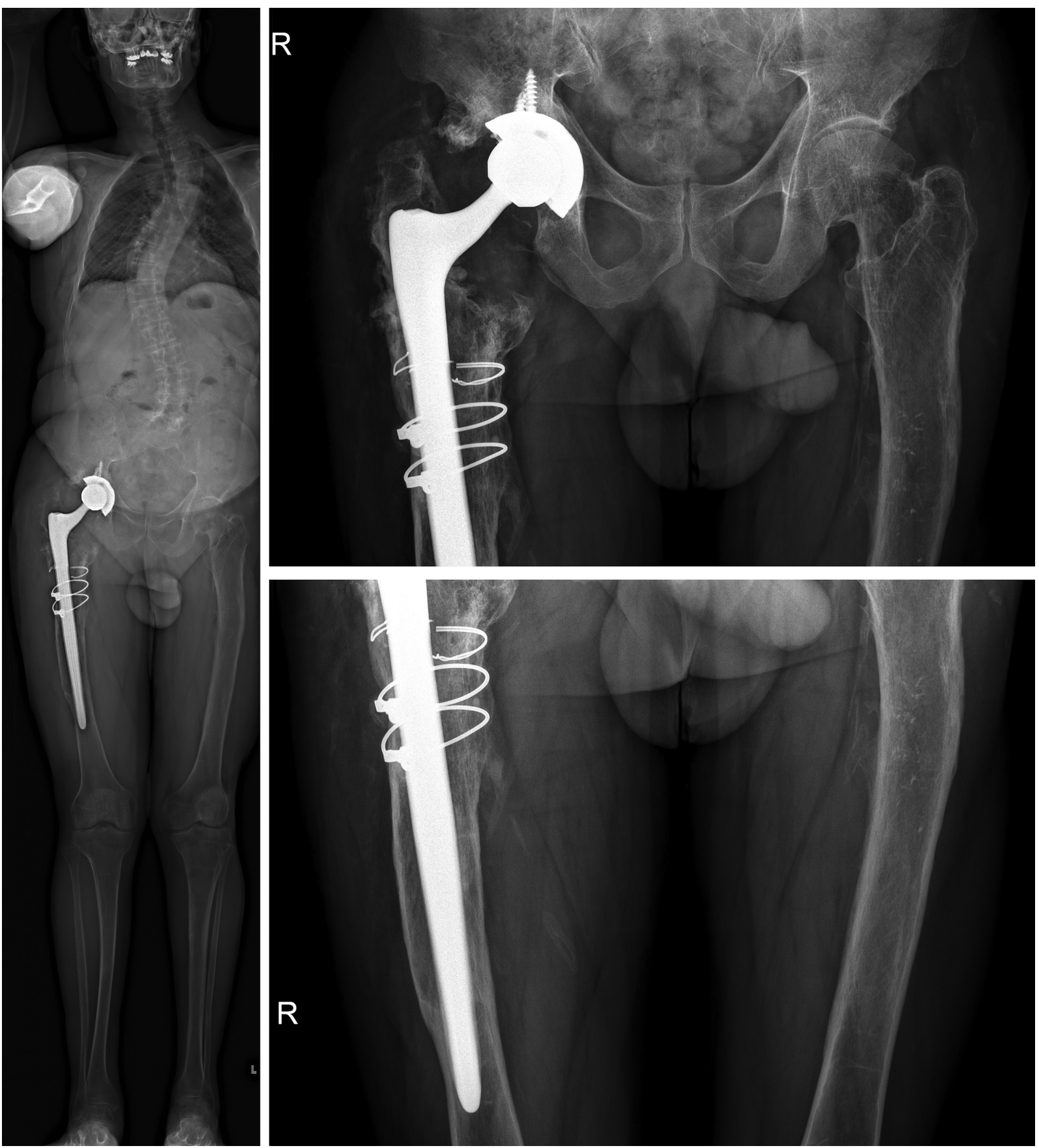

Fig. 3 Fourteen-month postoperative imaging. Lower extremity and anteroposterior pelvis radiographs demonstrating a well-fixed, wellpositioned right total hip arthroplasty.

with the use of noncemented stems. ${ }^{20}$ However, due to anatomical limitations, cemented stems may be difficult to centralize, preventing an adequate cement mantle from being placed around the prosthesis. ${ }^{11} \mathrm{~A}$ cementless, fluted tapered titanium monoblock stem was chosen for this case as the surgeon has vast experience using this stem in complex femoral reconstruction. Additionally, previous work has shown good outcomes in complex cases using monoblock stems. ${ }^{21-24}$ Regardless of implant type, precautions must be taken to limit intraoperative fractures.

Throughout this case, several precautions were taken to ensure a successful outcome and minimize the risk of intraoperative fracture. With adequate soft tissue release and exposure, the previous stem was safely extracted and a prophylactic wire was placed distally prior to reaming. Given the presence of a known pseudocanal, fluoroscopic imaging was used to assess placement of the guidewire, and flexible reamers were used to reestablish the femoral canal. A corrective osteotomy was also performed to avoid varus placement of the stem and an intraoperative fracture. Due to the increased risk of the procedure, final intraoperative fluoroscopy images and portable X-rays were taken and reviewed prior to completion of the operation to confirm that there were no fractures and the implant was correctly 
seated. Careful planning and the use of additional precautions may decrease the high rate of intraoperative fractures in these high-risk cases.

In addition to careful surgical planning, bone health is an important factor for the success of both primary and revision THA and should be addressed prior to surgical intervention. One suggested medical intervention to address bone quality is bisphosphonate therapy. While bisphosphonates do not address the underlying bone quality issues in OI, they do increase bone mineral density, decrease fracture rates, improve pain, and are a common medical treatment employed in this population. ${ }^{25-27}$ Studies on patients with osteoporosis have shown that bisphosphonates administered postoperatively are effective at reducing periprosthetic bone resorption in the first year after surgery, commonly seen after hip replacement. ${ }^{28-30}$ In a large retrospective cohort study in the Danish population, there was a $59 \%$ reduced risk of revision surgery if bisphosphonates were started after arthroplasty and continued for at least 1 year postoperatively. ${ }^{31}$ Use of bisphosphonates was also found to lower the fracture risk among THA patients who received the medication for at least 6 months prior to fracture, both as primary prevention and among patients who had experienced a previous osteoporotic fracture. ${ }^{32}$ Several additional studies have shown similar positive effects in patients taking bisphosphonates for a minimum of 6 months, which may persist as long as 18 to 72 months after discontinuation. ${ }^{33-36}$ While several studies have used a 6-month minimum duration of bisphosphonate treatment, the optimal timing and duration remain to be determined.

Although there is an initial decrease in the overall fracture risk with bisphosphonate therapy, with evidence to suggest improved bone mineral density and decreased revision rate after total joint arthroplasty, long-term therapy with these medications are associated with atypical femoral fractures, and patients need to be monitored carefully. ${ }^{37}$ In this case, the patient was taking ibandronate prior to his revision THA, though it was discontinued more than 1 year prior to surgery. Due to the rare incidence of the disease, no studies have looked at the effect of bisphosphonate therapy on joint arthroplasty in patients with OI, but there is potential benefit for use of this therapy in the surgical setting.

\section{Conclusion}

Improvements in both medical and surgical interventions in patients with OI have extended the life span of these patients and helped to improve quality of life. As THA is used more frequently in this population, further understanding of how these and other factors influence the likelihood of required THA revision will help ensure that the right patients are undergoing surgical intervention and the correct precautions are taken to minimize complications and decrease implant failure.

\section{Conflict of Interest}

Dr. Long reports personal fees from J\&J DePuy, ConvaTec,

Pacira, Think Surgical, Ortho Development, MicroPort, and TJO, outside the submitted work. Dr. Schwarzkopf reports grants and personal fees from Smith \& Nephew and Intellijoint, and personal fees from Gauss Surgical, outside the submitted work.

\section{References}

1 Tosi LL, Oetgen ME, Floor MK, et al. Initial report of the osteogenesis imperfecta adult natural history initiative. Orphanet J Rare Dis 2015; 10(01):146

2 Forlino A, Cabral WA, Barnes AM, Marini JC. New perspectives on osteogenesis imperfecta. Nat Rev Endocrinol 2011;7(09): 540-557

3 Rauch F, Glorieux FH. Osteogenesis imperfecta. Lancet 2004;363 (9418):1377-1385

4 Singer RB, Ogston SA, Paterson CR. Mortality in various types of osteogenesis imperfecta. J Insur Med 2001;33(03):216-220

5 Paterson CR, Ogston SA, Henry RM. Life expectancy in osteogenesis imperfecta. BMJ 1996;312(7027):351

6 Palomo T, Vilaça T, Lazaretti-Castro M. Osteogenesis imperfecta: diagnosis and treatment. Curr Opin Endocrinol Diabetes Obes 2017;24(06):381-388

7 Papagelopoulos PJ, Morrey BF. Hip and knee replacement in osteogenesis imperfecta. J Bone Joint Surg Am 1993;75(04):572-580

8 Marini JC, Forlino A, Bächinger HP, et al. Osteogenesis imperfecta. Nat Rev Dis Primers 2017;3:17052

9 Stevenson DA, Carey JC, Byrne JLB, Srisukhumbowornchai S, Feldkamp ML. Analysis of skeletal dysplasias in the Utah population. Am J Med Genet A 2012;158A(05):1046-1054

10 Moffitt KB, Abiri OO, Scheuerle AE, Langlois PH. Descriptive epidemiology of selected heritable birth defects in Texas. Birth Defects Res A Clin Mol Teratol 2011;91(12):990-994

11 Krishnan H, Patel NK, Skinner JA, et al. Primary and revision total hip arthroplasty in osteogenesis imperfecta. Hip Int 2013;23(03): 303-309

12 Roberts TT, Cepela DJ, Uhl RL, Lozman J. Orthopaedic considerations for the adult with osteogenesis imperfecta. J Am Acad Orthop Surg 2016;24(05):298-308

13 King JD, Bobechko WP. Osteogenesis imperfecta: an orthopaedic description and surgical review. J Bone Jt Surg 1971;53B(01): 72-89

14 Ahn J, Carter E, Raggio CL, Green DW. Acetabular protrusio in patients with osteogenesis imperfecta: risk factors and progression. J Pediatr Orthop 2017 (e-pub ahead of print). Doi: 10.1097/ bpo.0000000000001051

15 McBride MT, Muldoon MP, Santore RF, Trousdale RT, Wenger DR. Protrusio acetabuli: diagnosis and treatment. J Am Acad Orthop Surg 2001;9(02):79-88

16 Mullaji AB, Shetty GM. Acetabular protrusio: surgical technique of dealing with a problem in depth. Bone Joint J 2013;95-B(11, Suppl A ):37-40

17 Davidson D, Pike J, Garbuz D, Duncan CP, Masri BA. Intraoperative periprosthetic fractures during total hip arthroplasty. Evaluation and management. J Bone Joint Surg Am 2008;90(09):2000-2012

18 Berry DJ. Epidemiology: hip and knee. Orthop Clin North Am 1999;30(02):183-190

19 Bottai V, Dell'Osso G, Celli F, et al. Total hip replacement in osteoarthritis: the role of bone metabolism and its complications. Clin Cases Miner Bone Metab 2015;12(03):247-250

20 Mäkelä KT, Eskelinen A, Pulkkinen P, Paavolainen P, Remes V. Total hip arthroplasty for primary osteoarthritis in patients fifty-five years of age or older. An analysis of the Finnish arthroplasty registry. J Bone Joint Surg Am 2008;90(10):2160-2170

21 Sandiford NA, Garbuz DS, Masri BA, Duncan CP. Nonmodular tapered fluted titanium stems osseointegrate reliably at short term in revision THAs. Clin Orthop Relat Res 2017;475(01): 186-192 
22 Huang Y, Shao H, Zhou Y, Gu J, Tang H, Yang D. Femoral bone remodeling in revision total hip arthroplasty with use of modular compared with monoblock tapered fluted titanium stems: the role of stem length and stiffness. J Bone Joint Surg Am 2019;101 (06):531-538

23 Weeden SH, Paprosky WG. Minimal 11-year follow-up of extensively porous-coated stems in femoral revision total hip arthroplasty. J Arthroplasty 2002;17(04, Suppl 1):134-137

24 Herry Y, Viste A, Bothorel H, Desmarchelier R, Fessy MH. Long-term survivorship of a monoblock long cementless stem in revision total hip arthroplasty. Int Orthop 2019;43(10):2279-2284

25 Biggin A, Munns CF. Long-term bisphosphonate therapy in osteogenesis imperfecta. Curr Osteoporos Rep 2017;15(05):412-418

26 Dwan K, Phillipi CA, Steiner RD, Basel D. Bisphosphonate therapy for osteogenesis imperfecta. Cochrane Database Syst Rev 2014; (07):CD005088

27 Lozano-Calderon SA, Colman MW, Raskin KA, Hornicek FJ, Gebhardt M. Use of bisphosphonates in orthopedic surgery: pearls and pitfalls. Orthop Clin North Am 2014;45(03):403-416

28 Cavalli L, Brandi ML. Periprosthetic bone loss: diagnostic and therapeutic approaches. F1000 Res 2014;2:266

29 Muratore M, Quarta E, Quarta L, et al. Ibandronate and cementless total hip arthroplasty: densitometric measurement of periprosthetic bone mass and new therapeutic approach to the prevention of aseptic loosening. Clin Cases Miner Bone Metab 2012;9(01):50-55

30 Bhandari M, Bajammal S, Guyatt GH, et al. Effect of bisphosphonates on periprosthetic bone mineral density after total joint arthroplasty. A meta-analysis. J Bone Joint Surg Am 2005;87(02): 293-301

31 Prieto-Alhambra D, Lalmohamed A, Abrahamsen B, et al. Oral bisphosphonate use and total knee/hip implant survival: validation of results in an external population-based cohort. Arthritis Rheumatol 2014;66(11):3233-3240

32 Prieto-Alhambra D, Javaid MK, Judge A, et al. Fracture risk before and after total hip replacement in patients with osteoarthritis: potential benefits of bisphosphonate use. Arthritis Rheum 2011; 63(04):992-1001

33 Prieto-Alhambra D, Kassim Javaid M, Judge A, et al. Association between bisphosphonate use and implant survival after primary total arthroplasty of the knee or hip: population based retrospective cohort study. BMJ 2012;343:d7222

34 Lin T, Yan SG, Cai XZ, Ying ZM. Bisphosphonates for periprosthetic bone loss after joint arthroplasty: a meta-analysis of 14 randomized controlled trials. Osteoporos Int 2012;23(06):1823-1834

35 Khatod M, Inacio MCS, Dell RM, Bini SA, Paxton EW, Namba RS. Association of bisphosphonate use and risk of revision after THA: outcomes from a US Total Joint Replacement Registry. Clin Orthop Relat Res 2015;473(11):3412-3420

36 Teng S, Yi C, Krettek C, Jagodzinski M. Bisphosphonate use and risk of implant revision after total hip/knee arthroplasty: a metaanalysis of observational studies. PLoS One 2015;10(10): e0139927

37 Desai PA, Vyas PA, Lane JM. Atypical femoral fractures: a review of the literature. Curr Osteoporos Rep 2013;11(03):179-187 